\title{
Neuigkeiten aus der Romandie
}

Sandra Feroleto

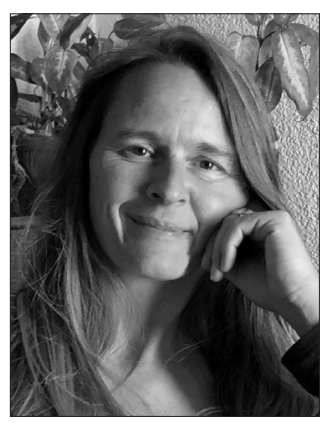

Mit dem Herbst kommen leichte Erkältungen und Viren halten Einzug, und das, wo COVID-19 immer noch wütet und in der Westschweiz sogar wieder einen Aufwärtstrend zeigt. Viele von Ihnen überdenken Ihre Praxis, haben sich mit Telefonkonsultationen und/oder Videokonferenzen neue Gewohnheiten angeeignet. Einige von Ihnen sehen sich daher angesichts der vom BAG auferlegten Einschränkungen mit Abrechnungsschwierigkeiten konfrontiert, die die Delegationsregelung mit sich bringt. Wir sind enttäuscht und bedauern, dass es uns nicht gelungen ist, bessere Bedingungen zu erhalten, die der aktuellen Realität der Bedürfnisse angepasst sind.

Dies besorgt uns umso mehr, da wir die allgemeine Beunruhigung in der Bevölkerung spüren, die aufgekommenen Ängste, die aussergewöhnlichen Reaktionen. Die menschliche Antwort auf diese einzigartige gesellschaftliche Situation ist polarisierend: Einerseits besinnen sich diejenigen, die Wärme und Humanismus im Dienste des Gegenübers betonen, wieder auf das Wesentliche, und andererseits reagieren diejenigen, die meinen, «ihre Haut retten zu müssen», auch wenn dies auf Kosten von Beziehungen erfolgt, auf eine Weise, wie sie es wahrscheinlich in keiner anderen Lebenssituation je getan hätten.

Wir werden in unseren Gewohnheiten erschüttert, mit unseren intimsten Ängsten konfrontiert, wir werden destabilisiert, auf den Prüfstand gestellt, aufgewühlt durch eine neue Realität, die wir begreifen müssen. Die Auswirkungen auf unsere Kinder und Jugendlichen sind besonders stark, und die Zahl der psychotherapeutischen Konsultationen, die von Studierenden an den Universitäten der Westschweiz beantragt werden, hat drastisch zugenommen.

Natürlich ist jeder auf andere Weise betroffen: Die Dienstleistungsberufe mussten ihre Arbeitsweise überdenken und/oder ihre Prioritäten neu ausrichten. Die digitalen Berufe boomen, und mehrere IT-Neugründungen in der Westschweiz haben ihren Umsatz in nur wenigen Monaten verdoppelt. Der Touristiksektor hat tiefgreifende Veränderungen erfahren. Reisen stehen nicht länger hoch im Kurs. Ihre ökologischen Auswirkungen sind zu stark und die drohenden Gesundheitsrisiken veranlassen viele Menschen zum Verzicht. Überraschenderweise haben unsere Berggaststätten so erfolgreich wie nie zuvor gearbeitet. Viele Menschen haben es vorgezo-

gen, aus den Städten zu fliehen und in die Ruhe der Berge zu flüchten, die glücklicherweise noch von der Maskenplicht verschont geblieben sind. Doch wie werden all diese kleinen und grossen Veränderungen die Menschen psychologisch beeinflussen? Die Kindertagesstätten in der Westschweiz machen sich Sorgen um unsere Kleinen, die kein Lächeln mehr sehen und nur noch einen eingeschränkten Eindruck von den Gesichtern haben, die seit Monaten hinter Masken verschwinden.

Manche Kolleginnen hören ihre kleinen Patient ${ }^{\star}$ innen sagen, dass sie für ihre Grosseltern gefährlich sind, dass sie sich ihnen nicht mehr nähern dürfen. Wie werden diese Kinder in der Lage sein, ihr Selbstwertgefühl zu entwickeln und mit einer generationenübergreifenden Dynamik aufzuwachsen, die in einem solchen Kontext Wärme, Weitergabe und Perspektive bringt?

Wir alle durchleben diesen Wandel der Rollenbilder - mit Vor- und Nachteilen. Nehmen wir das Beispiel des Homeoffice: Konnte ein Unternehmen Homeoffice bisher als Vorteil für seine Mitarbeiter*innen ansehen, so ist jetzt klar, dass sie vor allem ein Vorteil für das Unternehmen selbst darstellt, eine einfache Möglichkeit, den folgenschweren Auswirkungen wiederholter Quarantänemassnahmen zu entgehen.

Als Psychotherapeut ${ }^{\star}$ innen haben wir derzeit eine sehr wichtige Aufgabe zu erfüllen, einen Platz, den wir einnehmen müssen. Bei den Menschen, um sie zu beruhigen, um ihnen zu helfen, mit grösserer Gelassenheit auf die Zukunft zuzugehen. Um ihnen zu helfen, in Bewegung zu bleiben oder wieder in Bewegung zu kommen, das umzusetzen, was sie vermeintlich errungen haben, neue Wege zu beschreiten, die Herausforderungen, die diese einzigartige Zeit für sie bereithält, mit Zuversicht zu meistern. Aber wir haben auch eine politische Aufgabe zu erfüllen. Die Entscheidungen, die in diesem Krisenmanagement getroffen werden, müssen wir beeinflussen, indem wir auf die psychologischen Auswirkungen hinweisen, die diese Entscheidungen haben, auf den Einfluss, den diese oder jene Massnahme auf die Entwicklung eines Kindes, auf das Gleichgewicht eines Menschen nehmen kann.

In einer Zeit, in der die Krankenkassenprämien trotz eines gewissen Rückgangs der Pflegekosten wahrscheinlich steigen werden, wollen wir mehr denn je hoffen, dass der Bundesrat wirklich und 
schnell entschlossen ist, auf das Anordnungsmodell umzusteigen, das Psychotherapie endlich für den einfachen Bürger und die einfache Bürgerin zugänglich macht, der bzw. die heute leidet und sich Hilfe nicht leisten kann.

Die Westschweiz ist von COVID-19 besonders betroffen, und daher auch von den Massnahmen zu seiner Bekämpfung ... Hoffen wir, dass sie auch die Wiege einer gesunden psychotherapeutischen Innovation im Dienste der Generationen und der Welt von morgen sein wird ...

Sandra Feroleto ist Vorstandsmitglied der ASP und Delegierte für die Romandie.

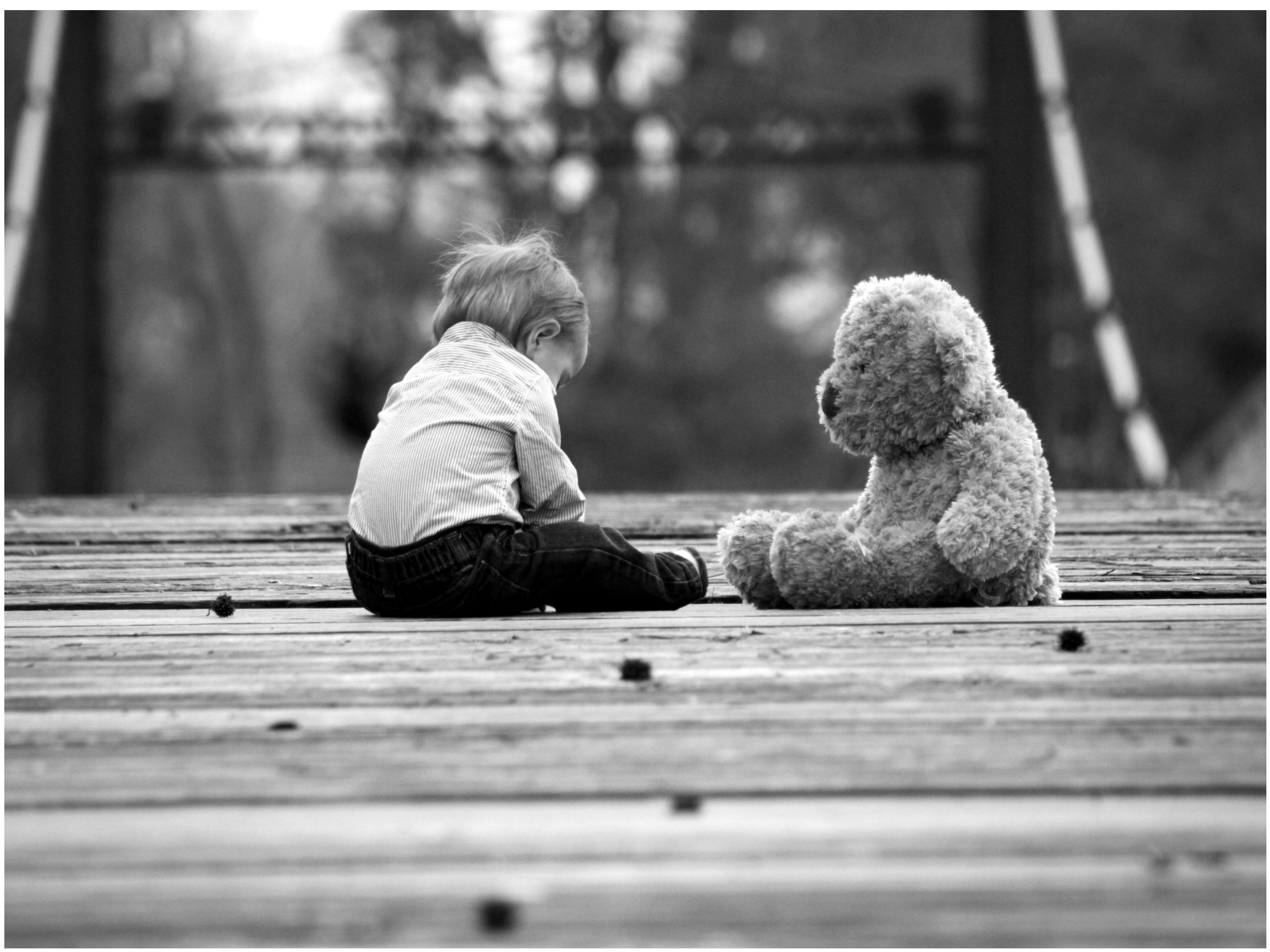

